\title{
Continuous Skyline Queries for Moving Objects in Road Networks
}

\author{
Changyue Shi ${ }^{1}$, Xiaolin Qin ${ }^{1 *}$, Li Wang1 \\ ${ }^{1}$ College of Computer Science and Technology, Nanjing University of Aeronautics and Astronautics, Nanjing, \\ Jiangsu, China. \\ *Corresponding author. Tel.: 86+13851642802; email: qinxcs@nuaa.edu.cn. \\ Manuscript submitted June 20, 2014; accepted August 30, 2014.
}

\begin{abstract}
Skyline queries in road networks have received much attention because of their wide applications in LBS. Due to the increasing demands for services, skyline queries for moving objects in road networks which involve not only static attributes, but also dynamic ones, become more important. This paper studies a problem of how to process the continuous skyline query for moving objects in road networks. We propose an event-based incremental processing algorithm on the strength of tracking dominant relationships between objects with the distances changing and finding the result changing points. Extensive experiments are conducted to evaluate the performance of our proposed algorithm.
\end{abstract}

Key words: Road networks, moving objects, skyline query, continuous query processing.

\section{Introduction}

In recent years, various location-based spatial queries have been proposed and researched, such as range queries, nearest neighbor queries [1], skyline queries [2] and so on. Skyline query is a kind of a kind of multi-criterion query. It retrieves the data objects which are not dominated by any other object. The skyline query in road networks involves both non-spatial attributes and the spatial ones, which fuels the demands of location-based applications, like traffic controlling, medical aiding, and advertising recommending.

The existing researches on skyline queries in road networks [3] mainly work on static objects, which have fixed locations in road networks. For example, users can check a skyline query to find out hotels nearby with cheaper price and more stars from many hotels. In this query, the distance between user and hotel is considered as a spatial attribute and the fixed price and stars are considered as non-spatial attributes.

However, dynamic traffic data bring challenges to the existing processing methods on static datasets. Many applications today begin to focus on moving objects in road networks. As an example shown in Fig.1, a man stands by the roadside waiting for a taxi nearby to serve him. In this scenario, we need to consider vehicle performance, driver's experience, user evaluation and the distance from him. In Fig.1, the taxis in solid line boxes $O_{1}, O_{2}, \ldots O_{5}$ show the locations of them at time $t_{1}$ and the ones in dotted boxes $O_{1}^{\prime}, O_{2}{ }^{\prime}, \ldots O^{\prime}$ are corresponding to their locations 30 seconds later. Considering their locations and non-spatial attributes listed in Table 1 , the skyline result set is $\left\{O_{1}, O_{5}\right\}$ at time $t_{1}$, and changes to $\left\{O_{1}, O_{2}, O_{3}, O_{5}\right\} 30$ seconds later. The distances from user to the taxis constantly change over time since the taxis are moving objects, and the skyline for the taxis is also changing. If we process the continuous skyline query for moving objects with repetitively snapshot queries, it will incur extremely high cost. 
Table 1. Non-Spatial Attributes of Taxis

\begin{tabular}{cccccc}
\hline \hline Taxi & $O_{1}$ & $O_{2}$ & $O_{3}$ & $O_{4}$ & $O_{5}$ \\
\hline Performance & 9 & 7 & 8 & 6 & 8 \\
Driver's Experience & 5 & 9 & 5 & 4 & 11 \\
User Evaluation & 9.4 & 8.9 & 9.2 & 9.0 & 9.0 \\
\hline \hline
\end{tabular}

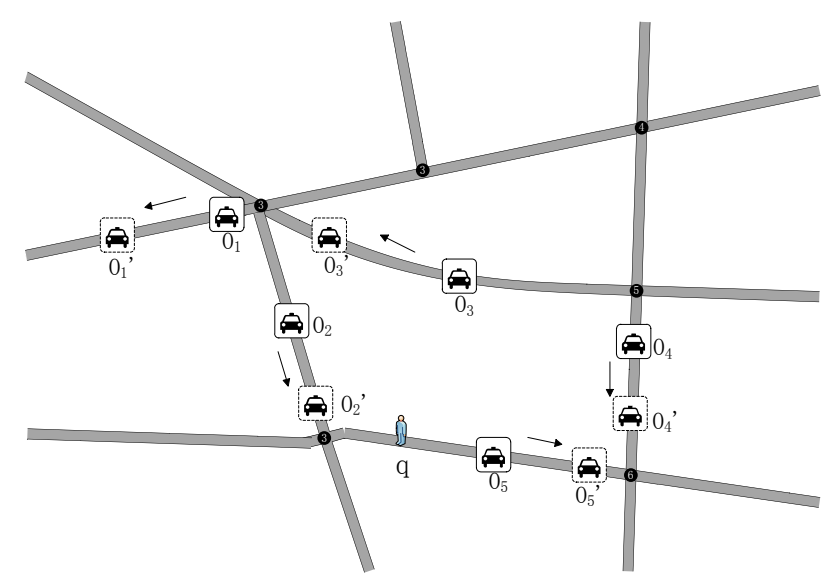

Fig. 1. Taxis' locations in a road network.

To solve the above issue, we study the continuous skyline query for moving objects in road networks and propose an efficient processing algorithm in this paper. We established explicit data models for items in the network environment and analyze the connection between distances of objects and their dominance relationships to find out the time points when the skyline result will change. Then we propose an event-based incremental algorithm to process the continuous skyline query for moving objects in road networks based on our models.

\section{Related Work}

The skyline query was first introduced by Borzonyi et al. as an operator in a relational database [4]. Two algorithms: Block-Nested-Loops (BNL) and Divide- and-Conquer (D\&C) were proposed and laid a foundation of skyline query processing. After that, a lot of scholars devoted to the researches on skyline queries, and successively put forward many variants of skyline query and corresponding efficient algorithms [5]-[7].

Deng et al. extended spatial skyline [8] to road network environment and studied the problem of multi-source skyline query (MSQ) processing in road networks [3]. They considered the distances from $m$ query points to the object as its $m$ dimensional attributes and proposed three pruning-based algorithms. Md. et al. studied a problem of $k$ dominant skyline for high dimensional space with a domination power index [9]. Huang et al. studied two variants of skyline query: continuous $d_{\varepsilon}$-skyline query $\left(\mathrm{Cd}_{\varepsilon}-\mathrm{SQ}\right)$ and continuous $k$ nearest neighbor skyline query (CKnn-SQ) [10]. They preferred the skyline points which were closer to the query point. Lin et al. studied the range-based skyline query in mobile environments for the reason of privacy protecting and the limited precision of positioning devices [11]. They put forward two algorithms: the index-base I-SKY algorithm and the non-index-based N-SKY algorithm. Mohammad et al. studied the skyline query for spatial objects by utilizing surrounding objects [12]. Cheema et al. studied a problem of monitoring a moving skyline query and proposed a safe zone based approach [13].

The studies mentioned above which focused on skyline queries over static datasets in road networks can't satisfy the increasing demands for location-based services. The collected data involving both static attributes and dynamic ones made the skyline queries for moving objects get more attention. Huang et al. did the first work on continuous skyline query in mobile environment and proposed the corresponding 
solution [14]. Tian et al. studied continuous monitoring skyline query over highly dynamic moving objects [15]. They divided the data space into influence region and free region. When some data changed, they updated the influence region and the skyline results dynamically. Later, Chen et al. [16] and Ding et al. [17] also did some researches on skyline queries for moving objects. However, these existing studies considered objects moving in a free space of 2 or more dimensions, or preset the objects with constant speeds on each dimension. Algorithms on these kinds of scenario assumption can't apply to the limited road networks. The directions and speeds of objects change constantly when they are moving along the roads. So a skyline query processing for such a set of moving objects will be more complex.

\section{Skyline for Moving Objects}

\subsection{Problem Definition}

Continuous queries for moving objects have extensive applications in LBS. In order to deal with the result changing, finding the changing points and updating the result will be more effective than snapshot processing in every timestamp. Before we describe the query processing in detail, we give the formal definition of this problem.

In this paper, we consider the query point $q$ as a static point and the data set $O$ as a set of moving objects in a road network. Each object $\mathrm{O}_{\mathrm{i}}=<\mathrm{S} 1, \mathrm{~S} 2 \ldots . . \mathrm{S} m \ldots \mathrm{S} n>$ in $O$ has $\mathrm{m}$ static non-spatial attributes and $n$ - $\mathrm{m}$ dynamic spatial ones. We consider the distance from query point as the only spatial attribute in this paper. For instance, in Fig. 1, the vehicle performance, driver's experience and the user evaluation are static non-spatial attributes because they are invariant. And the distance from query point varying continuously belongs to dynamic attribute.

Definition 1 (road-dominance). Given a query point $q$ and two moving objects $o, o^{\prime} \in O$, we say $o$ road-dominates $o^{\prime}$ if they met the following two conditions, and denote it as $O \prec_{q} O^{\prime}$.

1) For $1 \leq i \leq m, \forall i, o . S_{i} \leq \mathbf{0}^{\prime} S_{i} \wedge \exists i, o . S_{i}<0^{\prime} \cdot S_{i}$, it means $o$ non-spatially dominates $o^{\prime}$, denoted as $o \prec_{n s} o^{\prime}$;

2) $d(q, o)<d\left(q, 0^{\prime}\right)$, where $d(q, o)$ and $d\left(q, 0^{\prime}\right)$ represent the road distances of $o$ and $o^{\prime}$ from the query point $q$, respectively.

Skyline query for moving objects in road networks retrieves a subset of objects which are not road-dominated by any other object in $O$. It can be formally descripted as an object $o$ belongs to the result set if and only if there is no any object $o^{\prime} \in O$, s.t. $o^{\prime} \prec_{n s} o$ and $d\left(q, 0^{\prime}\right)<d(q, o)$.

Because of the locations of moving objects in the road network are changing with time, their distances from the static query point are also changing. A continuous skyline query for moving objects in the road network will retrieve the skyline objects constantly, referred as a MSkyline query for short.

\subsection{The Change of Skyline for Moving Objects}

According to the description in previous section, we know the MSkyline query involves both static non-spatial attributes and the dynamic distance. Some objects keep in the result set no matter where they are, and the others may enter or leave the result set with the distances changing. In this section, we will analyze the reasons which make skyline change to find the result changing points.

Theorem 1. For a moving object $o \in O$, if $o$ can't be non-spatially dominated by any other object in $O$, we say it is non-spatial dominator set $\operatorname{Dom}_{n s}(o)=\phi$. Then it will keep in the MSkyline result set, which is denoted as MSK for short.

Objects satisfying theorem 1. have outstanding non-spatial attributes which make them not road-dominated by others. We say these objects are fixed MSkyline objects, denoted them as permanent MSkyline set $M S K_{n s}$. Other objects belong to the candidate set $O_{\text {cand }}=O-M S K_{n s}$. 
Theorem 2. For a moving object $o \in O_{\text {cand }}$, whose $\operatorname{Dom}_{n s}(o) \neq \phi$, if $o$ is closer to $q$ than any other $o^{\prime} \in \operatorname{Dom}_{n s}(o)$, it will belong to the MSkyline result set $M S K$.

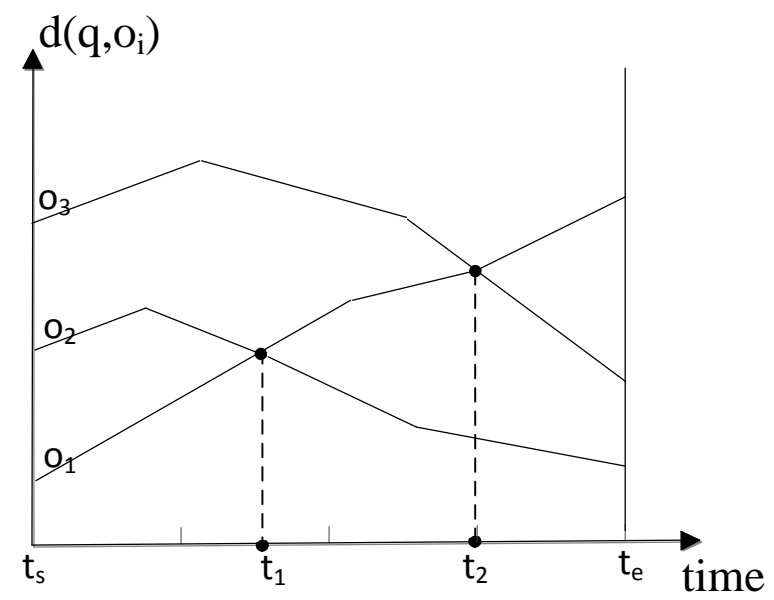

Fig. 2. Example of distance relationships.

Obviously, the distance relationships varying with the objects' moving may cause the MSK change. As an example of distance relationships shown in Fig. 2, three objects are moving with a constant distance relationship before time $t_{1}$ and the $M S K$ remains same during the time interval $\left(t_{s}, t_{1}\right)$. An intersection $\left(o_{1}, O_{2}, t_{1}\right)$ happens at time $t_{1}$ when the distance relationship between $o_{1}$ and $o_{2}$ changes from $d\left(q, o_{1}\right)<d\left(q, o_{2}\right)$ to $d\left(q, o_{2}\right)<d\left(q, \mathbf{o}_{1}\right)$. It may cause the object $o_{1}$ in $M S K$ to leave after time $t_{1}$, or the object oz not in $M S K$ to enter in. We analyze the following three possible cases to determine whether a distance intersection can make the MSK change.

Case 1: $o_{1} \notin \operatorname{Dom}_{n s}\left(\mathrm{O}_{2}\right) \wedge \mathrm{O}_{2} \notin \operatorname{Dom}_{n s}\left(\mathrm{O}_{1}\right)$

In this case, if there is no non-spatial dominance between $o_{1}$ and $o_{2}$, there will be no road-dominance either. The distance relationship between $o_{1}$ and $o_{2}$ will never affect the $M S K$.

Case 2: $\mathrm{O}_{1} \in \operatorname{Dom}_{n s}\left(\mathrm{O}_{2}\right) \wedge \mathrm{O}_{2} \notin \operatorname{Dom}_{n s}\left(\mathrm{O}_{1}\right)$

In this case, $o_{1} \prec_{n s} o_{2}$ and $d\left(q, o_{1}\right)<d\left(q, \mathbf{O}_{2}\right)$ Before time $t_{1}$. It means $o_{1}$ road-dominates $o_{2}$ which makes $o_{2}$ not belong to the $M S K$. When it arrives to time $t_{1}$, their distance relationship changes to $d\left(q, o_{2}\right)<d\left(q, \mathbf{O}_{1}\right)$. It makes $\mathbf{o}_{2}$ not be road-dominated by $o_{1}$ any more. If there is no other object in $\operatorname{Dom}_{n s}\left(\mathrm{O}_{2}\right)$ road-dominate $o_{2}, o_{2}$ will enter the $M S K$ after time $t_{1}$.

Case 3: $\mathrm{o}_{2} \in \operatorname{Dom}_{n s}\left(\mathrm{O}_{1}\right) \wedge \mathrm{O}_{1} \notin \operatorname{Dom}_{n s}\left(\mathrm{O}_{2}\right)$

In this case, the non-spatial dominant relationship between $o_{1}$ and $o_{2}$ is $o_{2} \prec_{n s} o_{1}$. When it arrives to time $t_{1}$, their distance relationship changes to $d\left(q, o_{2}\right)<d\left(q, 0_{1}\right)$. It makes $o_{2}$ begin to road-dominate $o_{1}$. If $o_{1}$ belongs to the $M S K$ before time $t_{1}$, it will leave. Otherwise, it will remain not in after time $t_{1}$.

\section{An Event-Based Algorithm for MSyline}

\subsection{Data Model}

To clarify the problem we study, we formally define the road network as an undirected weighted graph $G(V, E, W)$. It consists of a set of nodes $V$ and a set of edges $E$ which correspond to the road intersections and the road segments, respectively. The lengths of the edges are stored in $\mathrm{W}$. In addition, each edge also contains the following attributes shown in Table 2. 
Table 2. The Attributes of an Edge

\begin{tabular}{cc}
\hline \hline $\begin{array}{c}\text { Attributes } \\
\text { rid }\end{array}$ & Descriptions \\
eid & The identity of the road it belongs to \\
length & The edge identity \\
$n_{s}$ & The length of the edge \\
$n_{e}$ & The starting node of the edge \\
\hline \hline
\end{tabular}

To simplify the problem, we assume the query point as a static point in the road network and represent it as a Trituple $q=\left(q i d, e i d, d i s_{s}\right)$ where qid is the identity of $q$, eid is the identity of the edge which $q$ resides on, and diss is the distance between $q$ and the starting node $n_{s}$ of the edge. We consider a set of objects moving in the road network. The relevant attributes of moving objects are shown in Table 3.

Table 3. The Attributes of a Moving Object

\begin{tabular}{|c|c|}
\hline Attributes & Descriptions \\
\hline oid & The object identity \\
\hline eid & The identity of the edge it residing on \\
\hline$d i s_{s}$ & $\begin{array}{c}\text { The distance between the object and the } n_{s} \text { of the } \\
\text { edge it residing on }\end{array}$ \\
\hline$v$ & The moving speed of it \\
\hline$t_{u}$ & The nearest update time \\
\hline$t_{\text {next }}$ & The time it expected to reach the next node \\
\hline
\end{tabular}

We have the location information (eid,diss) and the speed $v$ of the object at update time $t_{u}$. A positive $v$ value indicates the object moves with the direction from $n_{s}$ to $n_{e}$ on the edge, and a negative $v$ value indicates it moves with the opposite direction. In addition, we use a vector $\operatorname{Dom}_{n s}(o)$ to manage the non-spatial dominators for each object $o$, with a tag $N_{u} m_{-} D_{o m}$ recording the number of the objects which are closer to $q$ than the $o$ bject $o$ in $\operatorname{Dom}_{n s}(o)$.

\subsection{Road Network Distance}

In order to deal with the continuous skyline query, the road distances between moving objects and the query point need to be constantly calculated. Although the Dijkstra's algorithm can be used to calculate distance between any two points in the network effectively, the constantly calculating with Dijkstra's algorithm will also bring enormous cost. For this reason, we design an optimize distance calculation method in road networks, which can greatly reduce the cost of constantly calculation.

The precomputed shortest distances between every two nodes $n_{i}$ and $n_{j}$ in the road network is denoted as $d\left(n_{i}, n_{j}\right)$ and stored in a distance matrix $S D$. The value of $d\left(n_{i}, n_{j}\right)$ in $S D$ can be used to promote the distance calculation between two objects on the edges linked with $n_{i}$ and $n_{j}$.
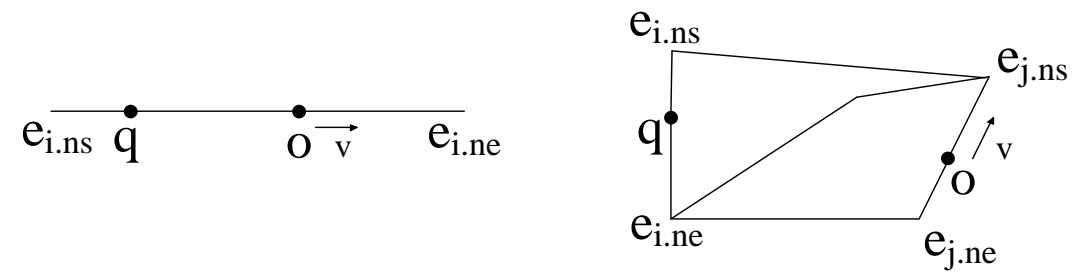

Fig. 3. Two cases of the locations between $q$ and $o$. 
Given a query point $q$ and a moving object $o$, the distance between them at time $t$, denoted as $d_{t}(q, o)$, can be calculated by our method with two cases shown in Fig. 3 based on their locations.

In the first case, they reside on the same edge $e_{i}$, and $o$ moves with the speed of $v$. We can calculate the distance between them by formula (1) as follows. The distance $d_{t}(q, o)$ obtained by the formula(1) is valid as long as $o$ is moving on $e_{i}$, and ensured changing in the range of [0, $e_{i}$ length].

$$
d_{t}(q, o)=\left|q . \operatorname{dis}_{s}-\left(o . d i s_{s}+o . v \times\left(t-o . t_{u}\right)\right)\right|
$$

In the second case, they reside on different edges $e_{i}$ and $e_{j}$. Since each edge has two nodes, the start node $n_{s}$ and the end node $n_{e}$, the distance $d_{t}(q, o)$ containing 3 parts can be calculated by formula (2), where $d\left(q . n_{s}, o . n_{s}\right), d\left(q . n_{s}, o . n_{e}\right), d\left(q . n_{e}, o . n_{s}\right)$ and $d\left(q . n_{e}, o . n_{e}\right)$ have been stored in $S D$.

$$
d_{t}(q, o)=\min \left\{\begin{array}{l}
d\left(q . n_{s}, o . n_{s}\right)+q . d i s_{s}+\left(o . d i s_{s}+o . v \times\left(t-o . t_{u}\right)\right), \\
d\left(q . n_{s}, o . n_{e}\right)+q . d i s_{s}+\left(e_{j} . l e n g t h-o . d i s_{s}-o . v \times\left(t-o . t_{u}\right)\right), \\
d\left(q . n_{e}, o . n_{s}\right)+\left(e_{i} . \text { length }- \text { q.diss }\right)+\left(o . d i s_{s}+o . v \times\left(t-o . t_{u}\right)\right), \\
d\left(q . n_{e}, o . n_{e}\right)+\left(e_{i} . \text { length }- \text { q.diss }\right)+\left(e_{j} . \text { length }-o . d i s_{s}-o . v \times\left(t-o . t_{u}\right)\right)
\end{array}\right\}
$$

Obviously, the distance $d_{t}(q, o)$ obtained by formula (2) keeps in the range of $\left[d_{\min }\left(q, e_{j}\right), d_{\min }\left(q, e_{j}\right)+e_{j} . l e n g t h\right]$ when $o$ is moving on $e_{j}$. Here $d_{\min }\left(q, e_{j}\right)$ refers to the distance along the shortest path from $q$ to $e_{i}$. Similar to the first case, when the object $o$ reaches the next node and move to another edge, the distance function will become invalid and need to be recalculated.

\subsection{The Algorithm}

Given a query point $q$ and a time interval $T$, the MSkyline query will return the continuous skyline set for a set of moving objects $O$ in the road network during the time period $\left[t_{0}, t_{0}+T\right]$. We propose an event-based algorithm $E_{-} M S K Y$ by tracking the valid events which will cause the $M S K$ change. For example, as shown in Fig.2, the distance intersection $\left(o_{1}, O_{2}, t_{1}\right)$ at time $t_{1}$ may change the dominant relationship between $o_{1}$ and $o_{2}$. We call this intersection an event. An event on $o_{i}$ and $o_{j}$ at time $t_{x}$ is denoted as event $\left(o_{i}, O_{j}\right)=<O_{i}, O_{j}, t_{x}>$. According to the analysis in section 3.2, not all distance intersections can bring change to the MSK. Therefore, we can use the following two pruning strategies to cut down the event set.

Pruning strategy 1: If there is no non-spatial dominance between $o_{i}$ and $o_{j}$, that is $o_{i} \notin \operatorname{Dom}_{n s}\left(o_{j}\right) \wedge o_{j} \notin \operatorname{Dom}_{n s}\left(o_{i}\right)$, the event $<o_{i}, o_{j}, t_{x}>$ is invalid to change the $M S K$, which can be pruned off.

Proof It is known that the static non-spatial attributes of $o_{i}$ and $o_{j}$ have no dominant relationship. No matter where they are moving in the road network, they can't road-dominate each other. The distance intersection between them can't cause any change to the MSK .

Pruning strategy 2: If $t_{x}>o_{i} . t_{n e x t}$ or $t_{x}>o_{j} . t_{n e x t}$, where $o_{i .} t_{n e x t}$ and $o_{j} . t_{n e x t}$ are the expected time $o_{i}$ and $o_{j}$ will reach the next node, the event $\left\langle O_{i}, \mathrm{o}_{j}, t_{x}>\right.$ is a wrong event which can be pruned off.

Proof Because of $t_{x}>o_{i} . t_{n e x t}$ or $t_{x}>o_{j} . t_{n e x t}$, which means the object $o_{i}$ or $o_{j}$ will reach the node and the distance function of $o_{i}$ or $o_{j}$ will has changed at time $t_{x}$, the event $<o_{i}, o_{j}, t_{x}>$ based on the previous distance function no longer exists.

In order to track the events accurately, we use a time queue $Q_{t}$ to store the times when moving objects will reach the next node and an event queue $Q_{e}$ to store the valid events in the order of the trigger time $t_{x}$. In the $E_{-} M S K Y$ algorithm, we firstly divide the time interval $\left[t_{0}, t_{0}+T\right]$ into many continuous and disjoint 
subintervals $\left(\left[t_{s_{1}}, t_{t_{1}}\right],\left[t_{s 2}, t_{e 2}\right], \ldots\left[t_{s n}, t_{e n}\right]\right)$ based on the time points in $Q_{t}$. Then trigger the events in these subintervals sequentially and update the result set $M S K$.We show the detailed query processing in algorithm 1.

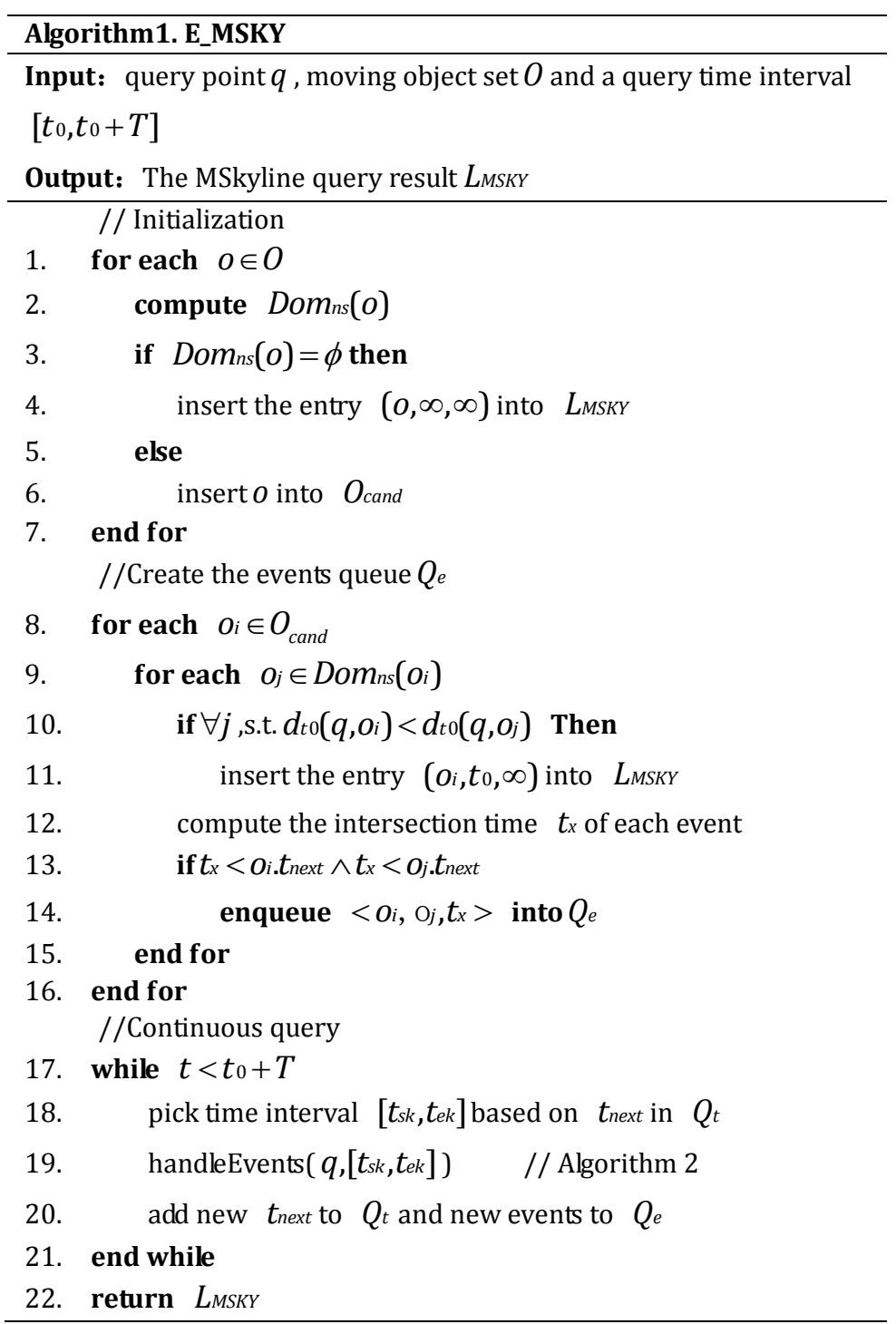

The initialization is the first step in $E_{-} M S K Y$ algorithm. Only considering the static non-spatial attributes, we can determine the non-spatial dominator set $\operatorname{Dom}_{n s}(o)$ for every object $o$ (line2), and distinguish the fixed MSkyline objects $M S K_{n s}$ which always belong to the result set (lines3-6).We implement the result set $M S K$ with a structure of linked list $L_{M S K Y}$. Each node in $L_{M S K Y}$ stores a Mskyline object and its entering and leaving time. All the initial Mskyline objects in addition to the fixed ones are added to LMSKY with the entering time $t_{0}$ (lines10-11). Then, the event queue $Q_{e}$ is initialized(lines13-14). After that, we divide the query time interval and handle the events during each subinterval sequentially(lines18-19). After each subinterval, there are some new time points and new events should be added to $Q_{t}$ and $Q_{e}$ (line20).

The events handling in each subinterval $\left[t_{s}, t_{e}\right]$ is presented as algorithm handleEvents in Algorithm 2. For a valid event $e<o_{i}, \mathrm{O}_{j}, t_{x}>\operatorname{in} Q_{e}$, in the case of $o_{i} \in \operatorname{Dom}_{n s}\left(o_{j}\right)$, we update the tag Num_Dom of object $o_{j}$. If there is no other object in $\operatorname{Dom}_{n s}\left(o_{j}\right)$ closer to $q$ than object $o_{j}$, add $o_{j}$ to $L_{M S K Y}$ with the entering time 
e.t. (lines3-6). In the other case of $o_{j} \in \operatorname{Dom}_{n s}\left(O_{i}\right)$, we update the tag Num_Dom $m_{d}$ of object $o_{i}$. If $o_{i}$ was a

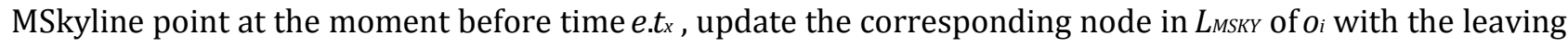
time e.t. (lines7-10).

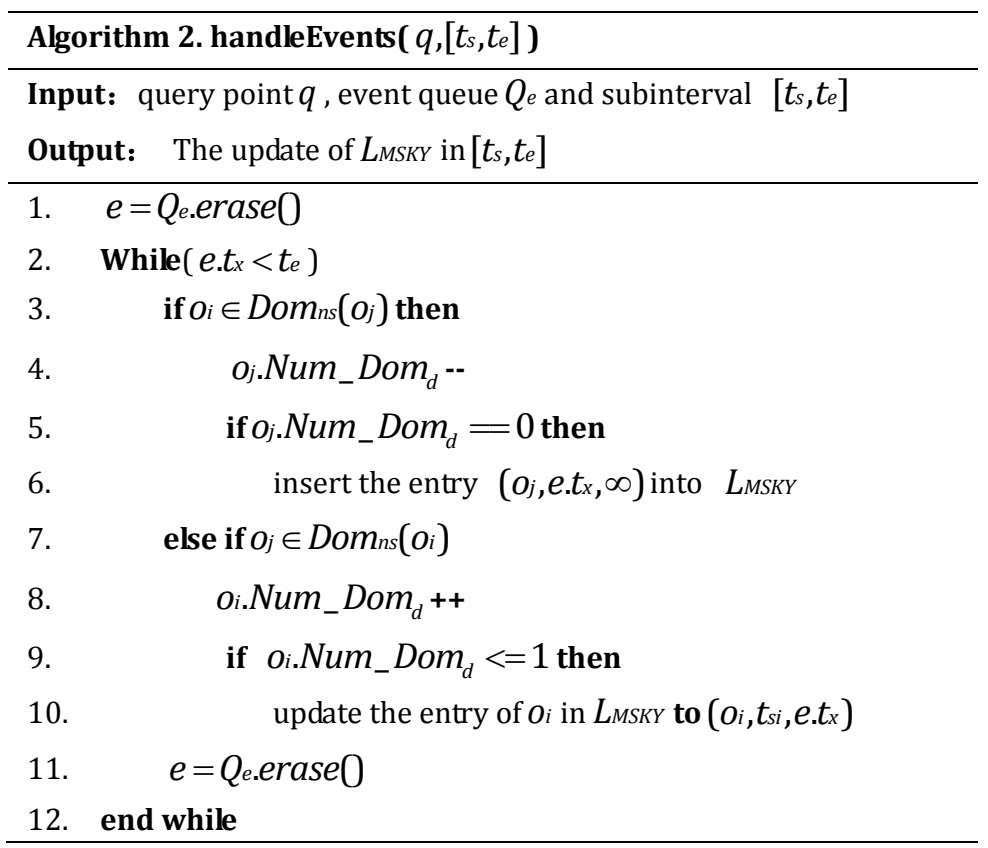

\section{Experimental Evaluation}

In our experiments, we consider a real network of Oldenburg in Germany [19], which contains 7035 road segments within the space territory of $23572 \mathrm{~m} \times 26915 \mathrm{~m}$. We use a generator extracted from [18] to generate moving objects in the road network. The relevant parameter settings are shown in Table 4 , the speed and the static attributes of objects randomly determined in the given ranges.

Table 4. Parameters Setting

\begin{tabular}{cc}
\hline \hline Parameter & Range \\
\hline Dataset cardinality & $20 \mathrm{~K}, 40 \mathrm{~K}, 60 \mathrm{~K}, 80 \mathrm{~K}, 100 \mathrm{~K}$ \\
Dimensionality of non-spatial attributes & $2,3,4,5,6$ \\
Range of non-spatial attribute value & {$[0,500]$} \\
\hline \hline
\end{tabular}

Since the continuous skyline query for moving objects studied by Huang et al in [12] is the closest study to this paper up to now. Although they assume the objects moving in the Euclidean space with a fixed speed in both directions, their algorithm CSQ also has a strategy for the updating of moving plan which can be used when the objects reach the road nodes in our network environment. So we conduct four sets of experiments to evaluate the performance of our $E_{-} M S K Y$ algorithm in contrast to $C S Q$ algorithm, running on Windows 7 operating system with $3.30 \mathrm{GHz}$ CPU and 2.0GB memory. In order to carry out a fair comparison, the query point is randomly generated and the queries in a comparison of two algorithms are based on the same data sets. What's more, the result is obtained based on averaging the 100 continuous queries for each set of data.

\subsection{Effect of Data Set Cardinality}

This set of experiments uses the data sets of moving objects including 3 non-spatial attributes and a distance 
attribute. The cardinality ranges from $20 \mathrm{~K}$ to $100 \mathrm{~K}$. As shown in Fig.4, we compare the CPU time and the event queue size at any time unit of two algorithms. As shown in Fig.4(b), the $E_{-} M S K Y$ has a bigger event queue size compared to $C S Q$, while it outperforms $C S Q$ in the CPU time cost shown as Fig.4(a). That is because the handling of each event in $E_{-} M S K Y$ is much simpler than the handling in $C S Q$.

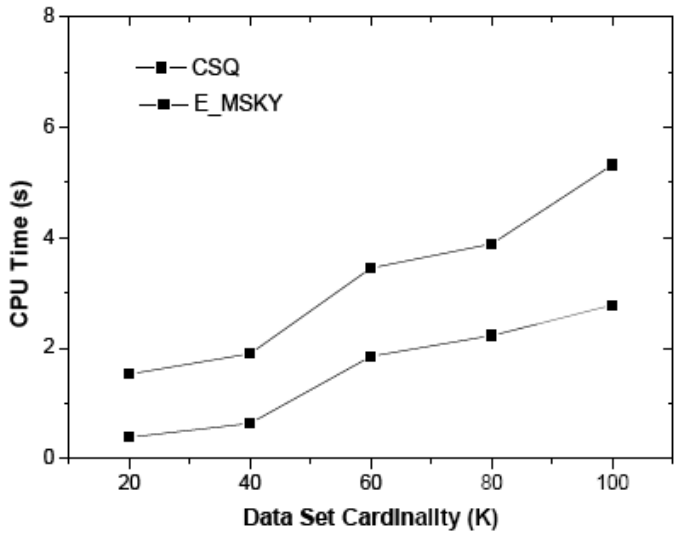

(a) CPU Time

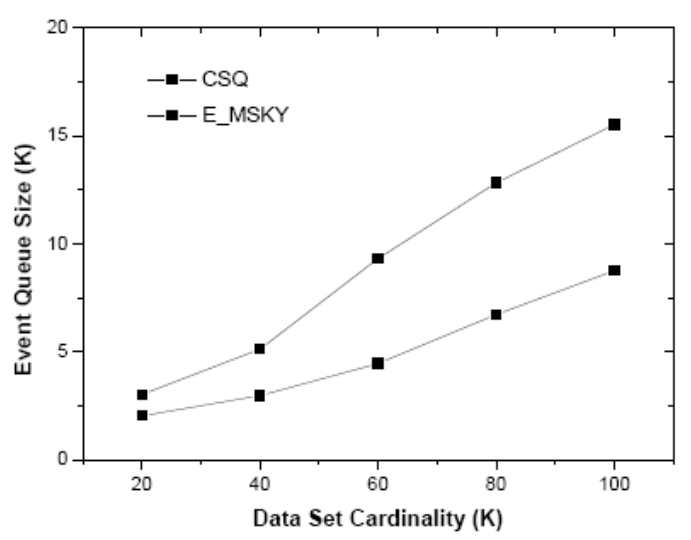

(b) Event Queue Size

Fig. 4. Effect of data set cardinality.

\subsection{Effect of Non-Spatial Dimensionality}

This set of experiments evaluates the performances on data sets of $50 \mathrm{~K}$ moving objects with the non-spatial dimensionality ranging from 2 to 6 . The results are shown in Fig. 5. Fig. 5(a) shows that the CPU time cost of $E_{-} M S K Y$ decreases with the increase of non-spatial dimensionality, which is opposite to CSQ and keeps much less than $C S Q$. The event queue size of $E_{-} M S K Y$ shown in Fig. 5(b) is also decreases with the increase of non-spatial dimensionality. When more non-spatial dimensionalities of the objects are considered, the possibility that an object can non-spatial dominate or be dominated by other objects will decrease, and there will be more fixed MSkyline objects and less dominators in $D_{0}$ of the candidate objects. This eventually reduces the number of events.

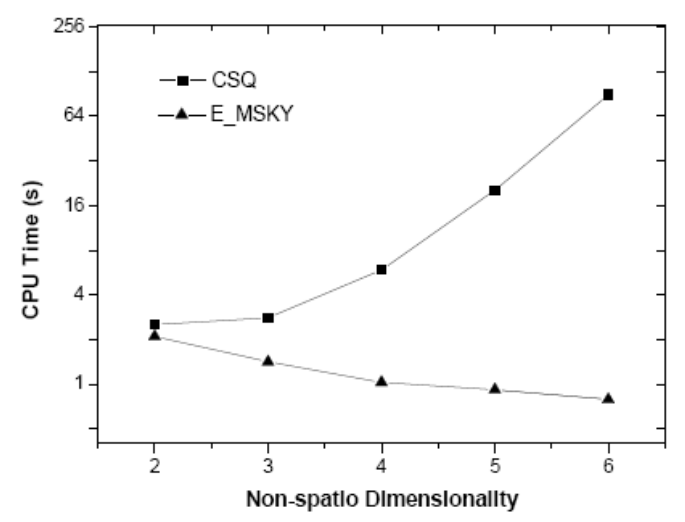

(a) CPU Time

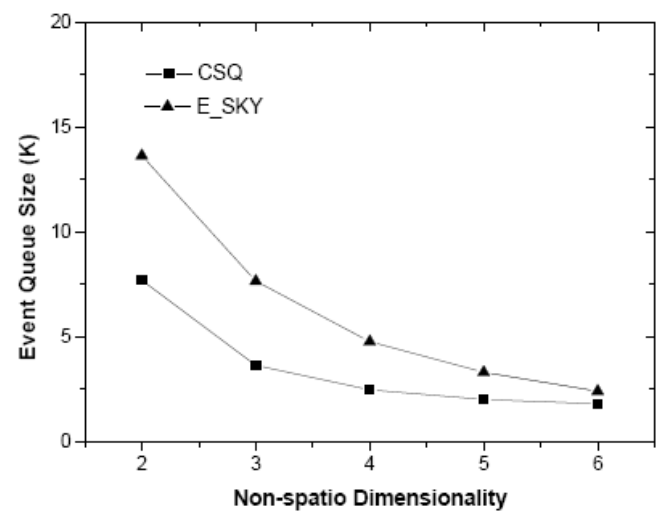

(b) Event Queue Size

Fig. 5. Effect of non-spatial dimensionality.

\subsection{Effect of Non-Spatial Dimensionality}

This set of experiments tests the effect of query time interval. We use a data set of 50K moving objects with 3 non-spatial attributes. The CPU time change with query time interval is shown in Fig. 6. With the query interval length increasing, the CPU time costs of both two algorithms are increasing. Obviously, more objects will reach the road nodes and move to another edge during a longer query interval, which will bring more events to handle 
and more update to the results.

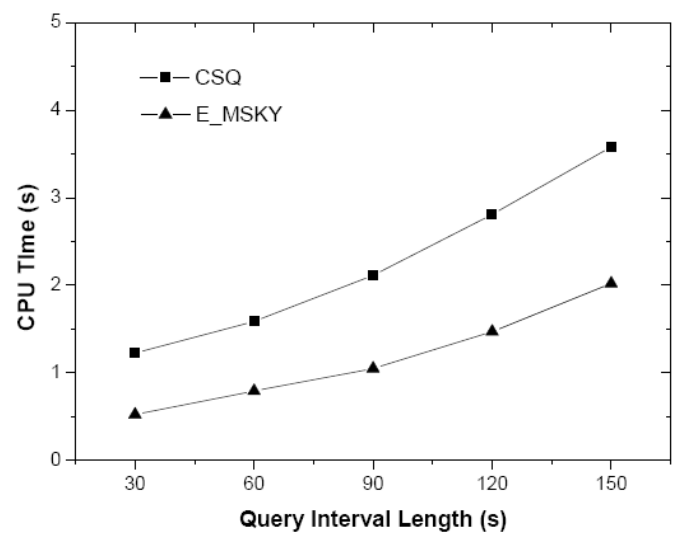

Fig. 5. Effect of query time interval.

\section{Conclusion and Future Work}

In this paper, we focused on a problem of efficient processing of continuous skyline queries for moving objects in road networks and proposed an event-based incremental algorithm for it. We fully considered the characteristics of the road network and moving objects for modeling and used result turning points to escape from snapshot queries. A lot of experiments we conducted and comparison with algorithm $C S Q$ in [14] have verified the superiority of our proposed algorithm.

In addition, the query point we considered in our problem is a static point, Therefore our future work will still focus on the skyline queries for moving objects in road networks, but with a moving query point.

\section{Acknowledgment}

This research was supported by the National Natural Science Foundation of China (Grant No. 61373015, 61300052) and the Research Fund for the Doctoral Program of High Education of China (Grant No. 20103218110017) . It was also supported by the Priority Academic Program Development of Higher Education Institutions in Jiangsu Province, China (PAPD) and the Fundamental Research Funds for the Central Universities, NUAA (Grant No. NP2013307).

\section{References}

[1] Fang, W.-K. (2013). Finding nearest neighbors in road networks: A tree decomposition method. Proceedings of the 16th International Conference on Extending Database Technology (EDBT).

[2] Lee, K. C.-K., et al. (2013). Efficient index-based approaches for skyline queries in location-based applications. IEEE Transactions on Knowledge and Data Engineering (TKDE), 25(11), 2507-2520.

[3] Deng, K., Zhou, X.-F., \& Shen, H.-T. (2007). Multi-source skyline query processing in road networks. Proceedings of the 23rd International Conference on Data Engineering (ICDE).

[4] Borzonyi, S., Kossmann, D., \& Stocker, K. (2001). The Skyline operator. Proceedings of the 17th International Conference on Data Engineering (ICDE).

[5] Kossmann, D., Ramsak, F., \& Rost, S. (2002). Shooting stars in the sky: An online algorithm for skyline queries. Proceedings of the 28th International Conference on Very Large Data Bases (VLDB).

[6] Papadias, D., et al. (2005). Progressive skyline computation in database systems. ACM Transactions on Database Systems (TODS), 30(1), 41-82.

[7] Chen, L., \& Lian, X. (2008). Dynamic skyline queries in metric spaces. Proceedings of the 11th International Conference on Extending Database Technology (EDBT): Advances in Database Technology, ACM.

[8] Sharifzadeh, M. \& Shahabi, C. (2006). The spatial skyline queries. Proceedings of the 32nd International Conference on Very Large Data Bases (VLDB). 
[9] Md., A.-S., \& Morimoto, Y. (2012). Efficient k-dominant skyline computation for high dimensional space with domination power index. Journal of Computers (JCP), 7(3), 608-615.

[10] Huang Y.-K., Chang C.-H., \& Chiang L. (2012). Continuous distance-based skyline queries in road networks. Information Systems(IS), 37(7), 611-633.

[11] Lin X., Xu J.-L., \& Hu H.-B. (2013). Range-based skyline queries in mobile environments. IEEE Transactions on Knowledge and Data Engineering(TKDE), 25(4), 835-849.

[12] Mohammad, S.-A., et al. (2013). Skyline query for selecting spatial objects by utilizing surrounding objects. Journal of Computers(JCP), 8(7), 1742-1749.

[13] Cheema, M.-A., et al. (2013). A safe zone based approach for monitoring moving skyline queries. Proceedings of the 16th International Conference on Extending Database Technology (EDBT).

[14] Huang, Z.-Y., et al. (2006). Continuous skyline queries for moving objects. IEEE Transactions on Knowledge and Data Engineering (TKDE), 18(12), 1645-1658.

[15] Tian, L., et al. (2007). “Continuous monitoring of skyline query over highly dynamic moving objects." Proceedings of the 6nd ACM International Workshop on Data Engineering for Wireless and Mobile Access (MobiDE).

[16] Chen, N., et al. (2012). PRISMO: Predictive skyline query processing over moving objects. Journal of Zhejiang University-Science C (JZUSC), 13(2), 99-117.

[17] Ding, X.-F., Jin, H., \& Xu, H. (2013). Probabilistic skyline queries over uncertain moving objects. Computing \& Informatics (CAI), 32(5), 987-1012.

[18] Brinkhoff, T. (2002). A framework for generating network-based moving objects. Geo Informatica, 6(2), 153-180.

[19] Census. Retrieved from http://www.census.gov/geo/www/tiger/

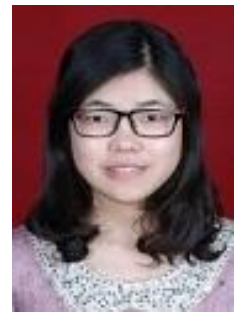

Changyue Shi was born in 1990. She is a master graduate student in Nanjing University of Aeronautics and Astronautics. She majors in computer science and technology and her current research interests are in the management of moving objects.

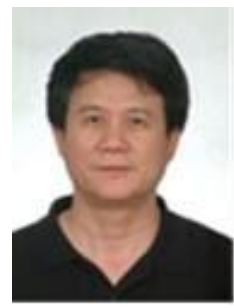

Xiaolin Qin was born in 1953. He is a professor and a PhD supervisor in Nanjing University of Aeronautics and Astronautics. He is a senior member of China Computer Federation. His main research interests include spatio-temporal databases, secure database, data management and disaster tolerance in distributed environment and information security.

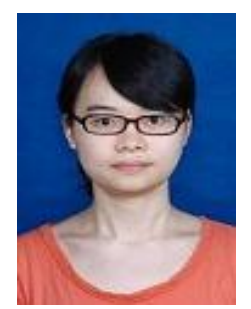

Li Wang was born in 1989, and she is a master graduate student in Nanjing University of Aeronautics and Astronautics. Her current research interests are in the management of indoor moving objects. 